\title{
Modern research and application analysis of rose flower
}

\author{
Feng Yu ${ }^{a}$, Miao Mingsan ${ }^{b, *}$ \\ Henan University of Chinese Medicine, Zhengzhou, China \\ aemail: feng000fengyu@163.com \\ bemail: miaomingsan@163.com
}

Keywords: Chinese rose, Chemical composition, Pharmacological effects, Application, essential oil.

\begin{abstract}
The Chinese rose flower contains flavonoids, volatile oil and other effective parts. It has anti - aging, anti - oxidation, anti - sugar, inhibition of tumor and other pharmacological effects. Chinese rose flower as medicine and food medicine clinical in gynecological diseases, carbuncle swelling, traumatic pain, diabetes treatment has a better curative effect. The therapeutic effect is also obvious. There is also a good space in the skin care market. The flower is rich in resources and cheap. This paper summarizes the modern research and application of the rose flower in order to promote the development of the monthly flower industry.
\end{abstract}

\section{Introduction}

Chinese Rose flower is the flower of Chinese Rose, alias monthly rose. This product started in the Ming dynasty li shizhen's "compendium of materia medica", and the "rose flower" for the record, has been used to this day. Rose flowers are sweet and warm, and they are aromatic, slightly bitter, and diffuse into the liver. Both blood and Qi, Jieyu regulate menstruation, swelling and detoxification. Treatment of blood stagnation and liver qi with the use of treatment of swelling and injury can be cast. It is used for bruising, menstruation, menopause, etc. Rosa chinensis has an important position in the study of modern blood-activating and stasis-removing drugs. We found that a kind of tea bag prepared by Rosa chinensis and Astragalus membranaceus can also effectively treat diabetes mellitus ${ }^{[1]}$. Total flavonoids of Rosa chinensis can be used to prepare hypoglycemic drugs effectively ${ }^{[2]}$, and can also significantly improve brain injury, energy metabolism and inflammatory factors in cerebral ischemia-reperfusion model mice ${ }^{[3]}$. Modern studies have shown that Chinese rose has a variety of chemical constituents such as quercetin, volatile oil, and has a variety of pharmacological effects on uterine smooth muscle excitation, bacteriostasis and so on. In addition, many scholars have used the extracts of the rose flower to prepare the antipruritic ointment ${ }^{[4]}$.Use the rose extract to make the mask liquid ${ }^{[5]}$ and the shower Gel etc ${ }^{[6]}$.This indicates that the rose flower has important medicinal value and research and development value.

\section{Major chemical components}

\section{The essential oil}

There are obvious similarities and differences between the volatile oils in rose and Chinese rose. $\mathrm{Li} \mathrm{fei}{ }^{[7]}$ analyzed the composition of the volatile oil of rose and Chinese rose flower with the use of temperament association, and found that there were 22 components of the volatile oil of Chinese rose. There are 21 types of volatile oil in Chinese rose. These common groups are divided into alkanes or ene alkane. The amount of volatile oil in the rose is higher than the Chinese rose.

\section{Trace elements}

Using atomic spectrophotometry to determine the contents of four trace elements of copper, zinc, magnesium and calcium in the Chinese rose, the content of calcium and zinc is very rich, which is 


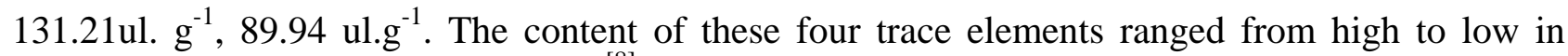
calcium, zinc, magnesium and copper ${ }^{[8]}$.

\section{Flavonoids, Phenolic acids, Sterols, etc.}

Zhao Qian ${ }^{[9]}$ found that there were 14 kinds of phenolic acids and flavonoids in the ethyl acetate layer and n-butanol layer of dry flower..Wang Xiaoyan ${ }^{[10]}$ obtained 15 compounds from dried rose flowers. Among them, only the kaempferol -3-O- $\beta$-D- glucoside was found in the same way as Zhao Qian et al.Cai Yuanyuan ${ }^{[11]}$ also obtained 11 identified monomer compounds from the nbutanol part of Chinese rose flowers including tannic acid,saponins $\beta$-glucoside, ursolic acid and other triterpenoids, flavonoids.

\section{Pharmacological effects}

\section{Anti-oxidation, anti-fatigue and anti-aging}

Four flavonoids from the dried rose flowers have the ability to remove DPPH•, among which ethanol-ethyl ester and n-butanol extract have a good effect in removing DPPH•, while petroleum ether extract has a poor effect in removing free radicals ${ }^{[12]}$. After the mice were given the laurium pigment for 4 weeks, the swimming time of the mice was significantly prolonged, and the activity of various antioxidant enzymes in the body was also enhanced, and the sense of physical fatigue was delayed ${ }^{[13]}$.In addition, wen jianfei ${ }^{[14]}$ found that the exercise oxidative stress was relieved after mice were given moonflower pigment.

\section{Reduce the blood sugarTrace elements}

Chinese rose flavonoids is the effective component of hypoglycemic experiment ${ }^{[15]}$, the adrenaline, caused by a chain of urea with cephalosporins type 1 diabetes and diabetic nephropathy model has a better therapeutic effect, Chinese rose flavonoids may by promoting synthesis of hepatic glycogen, inhibition of sugar dysplasia, promote the secretion of insulin to lower blood sugar, but also by improving lipid metabolism and so on ways to improve organ pathological changes, and delay the development of diabetes.

\section{Contraction smooth muscle}

The water decoction of Chinese rose flower can significantly inhibit the spontaneous contraction amplitude and contraction frequency of isolated uterus of mice,antagonistic uterine excitations caused by oxytocin, and show a certain dose-dependence ${ }^{[16]}$.

\section{The application of Chinese rose flower}

\section{Clinical application}

\section{Gynecological diseases}

In the control treatment of 49 patients with ovulation disorders, it was found that the indicators of ovulation rate and endometrial thickness of the control group were significantly better than those of the treatment group after taking compound prescriptions composed of radix bupleuri, yu jin, Chinese rose, safflower and salvia miltiorrhiza ${ }^{[17]}$. Professor han bing often USES zhibai dihuang pill together with blood-activating drug chuanxiong and Chinese rose flower to treat the febrile polycystic ovary syndrome of Yin deficiency syndrome ${ }^{[18]}$. Yu zhaoling ${ }^{[19]}$ believed that although rose flowers have similar efficacy with roses, they are lighter in quality, clearer in gas and more suitable for promoting ovulation discharge. Therefore, rose flowers are often used to treat dysmenorrhea, breast lumps, white spots in vulva, ovulation disorders, hair loss and other diseases.

\section{The upper disease}

"Upper disease" refers to diseases on the face and body surface caused by hot blood, such as wine trough nose, chloasma, acne, etc. Han shirong used flocculus decoction "red flower, Chinese 
rose, rose, ling xiao flower, chrysanthemum" to treat pigmentation. He believed that flower medicinally clean and clear can be applied to the skin, which is very suitable for the treatment of chloasma. Chinese rose flowers bloom every month, which is consistent with female menstruation. Therefore, Chinese rose flowers can regulate menstrual freckles ${ }^{[20]}$. The combination of Chinese rose, codonopsis, honeysuckle and other flower drugs can also be used to treat "upper diseases" such as erythema stage of disk lupus and pityriasis of rose.

\section{Others}

The carbuncle swelling can be treated by smashing or grinding the fresh Chinese rose flower. Treatment of pulmonary deficiency cough hemoptysis with $10 \mathrm{~g}$ of Chinese rose and rock candy stews; Rose stewed red wine can treat postpartum Yin ting. Some studies reported that daily boiling water brewed fresh rose flower $30 \mathrm{~g}$, frequent drinking, long-term drinking after the chest tightness, chest pain and other hidden coronary heart disease symptoms can be reduced. Chinese rose flower, ground turtle insect equal amount grind fine end, each time $5 \mathrm{~g}$, everyday 2 times, warm wine takes a little, use flower or leaf pound additionally apply affected part to be able to treat traumatic swelling pain.

\section{Therapeutic application}

\section{Chinese rose flower porridge}

Boil 100g japonica rice into porridge. Add 5 Chinese rose flowers and 100 brown sugar $^{[21]}$. Chinese rose, trumpetflower and japonica rice were collected in proportion of 1:1:5 and ground into fine powder. After boiled into porridge with japonica rice, the mixture of Chinese rose roller xiao powder and brown sugar was added to form Chinese rose roller xiao porridge.

\section{Chinese rose tofu}

Take 2 Chinese roses, 500g tofu, 1 cooked salted duck egg, 5 g curry powder, 50g vegetable oil and mustard juice, appropriate amount of cucumber, white vinegar and salt. Wash and shred the Chinese roses, blanch the tofu, cut the salted duck eggs and cucumber into small pieces, puree the egg yolk with the tofu, boil the mustard juice and mix the allene with white vinegar. The curry powder is stir-fried with hot oil. Add the prepared ingredients, stir well and put on the plate. This diet formula is delicious and tasty, which can strengthen spleen and invigorate qi, invigorate blood and regulate meridians.

\section{Application of rose essential oil}

The intense fragrance of rose essential oil is the main source of rose essence, is one of the main fragrance of plain orchid perfume, and is also the main raw material of modern 100-flower fragrance. Rose essential oil can be used in the deployment of a variety of edible flavors, bath soap, after the use of skin supple, delicate, also can be used as a natural air purifier. Wang xiaowei et al. invented a solid mosquito repellent containing essential oil of Chinese roses, effective components of camphor leaves, ethanol and other components, which can drive mosquitoes and clean air well ${ }^{[22]}$.

\section{The rose flower external use protects skin}

Due to the pharmacological effects of the anti-oxidation and anti-aging of Chinese rose, in recent years, it is quite popular to make Chinese rose extract into moisturizing skin care products. Many individuals or enterprises have applied many patents for skin care products for external use of Chinese rose. Zhu wei invented a kind of antipruritic cream that can be used after a baby is bitten by mosquito. The baby's delicate skin can adapt to this product, which can effectively relieve the swelling after the bite ${ }^{[4]}$. Wang yifei ${ }^{[23]}$ disclosed a moisturizing skin care product containing mangosteen extract, Chinese rose extract and blueberry extract, believing that this product is especially suitable for sensitive skin and can maintain skin elasticity and smoothness. Chen haijia ${ }^{[24]}$ invented a facial mask cream and preparation method mainly containing Chinese rose ultra 
powder and Chinese rose extract. The secondary skin care products have good stability, and have a moisturizing and tender effect on the skin.

\section{Prospects}

Chinese rose is native to China. It has been cultivated for more than 2,000 years. Nowadays, Chinese rose has become the flower of 52 cities such as Beijing. The rose blooms every month. Compared with the rose, the rose has natural advantages in clinical medication due to its rich resources, cheap price and easy access. In addition, the leaves and roots of Chinese rose can also be used as medicine. In hunan medical records, Chinese rose leaves were recorded as medicine name for the first time. It can treat the pain of bones and muscles, lumbar and knee swelling, wound rupture, hemostasis and swelling. The root of Chinese rose as medicine name appears for the first time in "fujian east herbage", add a few Chinese rose root when stewing crucian carp to be able to treat the scrofula scrofula unbroken; $30 \mathrm{~g}$ of Chinese rose root, $250 \mathrm{~g}$ of pork, stewed to cure blood spasm; Chinese rose root one or two, cockscomb one or two, motherwort three money, Fried water stewed egg can treat menstrual pain. But the Chinese rose often USES the flower medicine, has caused the resource waste.

As an important blood circulation drug, Chinese rose has good curative effect in polycystic ovary syndrome, dysmenorrhea, chloasma, ovulation disorders and other diseases. However, according to the clinical application of rose flowers, the external use of rose flowers is less, and under the guidance of the theory of traditional Chinese medicine, the external function of rose flowers should be developed to expand the application range of rose flowers.

Because the ingredients, such as Chinese rose contain flavonoids, volatile oil in oil research as well as cosmetics, skin care products and so on have the larger development space and the comprehensive utilization value, but for the details of Chinese rose essential oil composition and safety assessment of skin care products is less, in order to make Chinese rose are fully utilization of resources, expand the scope of product sales, get more consumer trust, dealing with the specific role position of the Chinese rose essential oil, mechanism of action of system research, guide the development of Chinese rose skin care products combined with traditional Chinese medicine, make skin care products market more specification.

\section{Acknowledgements}

Fund projects: National International Cooperation Base (2016-151), Central Plains Scholars (162101510003), Zhengzhou Science and Technology Research (131PPTGG417-3), Zhengzhou International Cooperation (141PGJHZ545)

First author: Feng Yu, Pharmacology. Email: feng000fengyu@163.com.

Correspondence Author: Miao Mingsan, male, Professor, doctor. Mainly engaged in pharmacology teaching and research of Chinese medicine. Email: miaomingsan@163.com.

\section{References}

[1] Miao mingsan, bai li, miao yanyan. A kind of Chinese rose flower composite bag for diabetes treatment [P]. Henan: CN106266059A, 2017-01-04.

[2] Miao mingsan, wang can, qiao jingyi. A method for extracting glucose-lowering substances from Chinese rose flowers and its application [P].Henan: CN103463244A, 2013-12-25.

[3] Miao mingsan, fang xiaoyan. A method for the extraction of anti-cerebral ischemic substances from Chinese roses and its application [P]. Henan: CN103432240A,2013-12-11.

[4] Zhu wei.An antipruritic cream for infants with extract of Chinese rose flowers after mosquito bites [P]. Guangdong: CN106266048A,2017-01-04.

[5] Xie caihong.A Chinese rose essence facial mask [P].Guangdong: CN106726955A,2017-05-31. 
[6] Yin haiquan. A kind of tightly-firmed shower gel of Chinese rose and its preparation method [P]. Heilongjiang: CN107049909A, 2017-08-18.

[7] Li fei, Yang yuanxia. Comparison of volatile oil components between rose and Chinese rose [J]. China Pharmacist,2016,19(01):182-184.

[8] Jin lie, ma jiao li. Determination of trace elements in scented tea by atomic absorption spectrophotometry [J]. Guangdong Chemical Industry, 2017,44(15):259-260+249.

[9] Zhao qian, liu francium, li qingjuan, et al. Study on the chemical constituents of Chinese rose flowers [J]. Chinese Traditional and Herbal Drugs, 2012,43(08):1484-1488.

[10] Wang xiaoyan, wang wenwen, zhou yonghui,et al. Preliminary study on the chemical constituents of Chinese rose flowers[J]. Chinese Pharmaceutical Journal,2012,47(07):500-503.

[11] Cai yuanyuan. Study on the chemical composition and anti-tumor activity of n-butanol in rose flower [D]. Zhengzhou university,2014.

[12] Wang lei, fu ling, jing linlin, et al. Studies on the antioxidant active components of Chinese rose flowers [J]. Journal of chemistry,2012,33(11):2457-2461.

[13] Yuan kexing, wang haitao, chang lixin, et al. Effects of Chinese rose pigment on antioxidant enzyme system and lactic acid content in sports mice [J]. Journal of Hebei Normal University (Natural Science Edition),2011,35(05):515-518+531.

[14] Wen jianfei, wang qiang, yu yonghao, et al. Effects of Chinese rose pigment on oxidative stress damage of skeletal muscle of exercise rats [J]. Liaoning Sport Science and Technology,2013,35(04):24-25+66.

[15] Hou chaojun. Effects of total flavonoids of Chinese rose flowers on diabetic models in mice and rats [D]. Henan college of Chinese medicine,2014.

[16] Liang huayi, han guangshun, huang chunjia, et al. Effect of Chinese rose extract on contractility of isolated uterine smooth muscle of mice [J]. Maternal and Child Health Care of China, 2018,33(01):182-184.

[17] Li xiahua, xu huiqun, tong xuefei. Observation on the curative effect of kidney, liver and blood stasis therapy on 49 cases of ovulation disorders [J]. Journal of New Chinese Medicine, 2014, 46(06):145-147.

[18] Dai zhirong, li peilin. Experience of han bing from treating polycystic ovary syndrome by phase-fire [J]. Shanxi Journal of Traditional Chinese Medicine, 2014, 30(01):10-11.

[19] Liu wen-e, Lin jie, Chen yan-xia, et al. Summary of professor yu zhaoling's experience in the application of flower drugs [J]. China Journal of Traditional Chinese Medicine and Pharmacy, 2014,29(06):1866-1868.

[20] Li meihong, li ning, han shirong. Han shirong's experience in treating chloasma [J]. Shaanxi Journal of Traditional Chinese Medicine, 2016,37(08):1064-1065.

[21] Five types of traditional Chinese medicine cosmetic congee.[J] Hunan Journal of Traditional Chinese Medicine,2016,32(09):78.

[22] Wang xiaowei.A solid repellent and its preparation method: China, CN201410442653.5 [P].2015-01-07.

[23] Wang yifei, ge xiaohu, Chen haijia,et al. A moisturizing and soothing skin care product and its preparation method [P]. Guangdong: CN107753341A, 2018-03-06.

[24] Chen haijia, ge xiaohu, wang yifei,et al. Chinese rose mask cream and preparation methods [P]. Guangdong: CN107349172A, 2017-11-17. 